\title{
OBSTÁCULOS ORGANIZACIONALES Y CULTURALES QUE LIMITAN LA TRANSFERENCIA DEL CONOCIMIENTO EN LA UNIVERSIDAD AUTÓNOMA DE QUERÉTARO'
}

\section{ORGANIZATIONAL AND CULTURAL BARRIERS THAT LIMIT THE KNOWLEDGE TRANSFER KNOWLEDGE AT THE AUTONOMOUS UNIVERSITY OF QUERETARO}

\author{
Rosa María Romero González ${ }^{2}$ \\ Universidad Autónoma de Querétaro
}

RECIBIDO: Septiembre 02 de 2016

ACEPTADO: Noviembre 30 de 2016

\begin{abstract}
RESUMEN
El objetivo principal de este trabajo fue identificar cómo afectan los obstáculos organizacionales y culturales durante la transferencia del conocimiento en la Facultad de Informática de la Universidad Autónoma de Querétaro (UAQ). Se realizó un análisis teórico sobre aprendizaje organizacional, su importancia e impacto para la transferencia del conocimiento. Se aplicó un cuestionario con 126 reactivos en escala Likert a una muestra de 236 alumnos de nivel licenciatura. Se obtuvo el coeficiente Alfa de Cronbach de .960 indicando una alta consistencia interna entre los ítems. Se efectuó el análisis factorial confirmatorio para determinar el efecto de las variables latentes sobre las variables observadas. Por tanto, se observó que organizacionalmente, se fomenta el aprendizaje y la creatividad en las actividades educativas, introduciendo constantemente nuevas ideas. Por la parte cultural, los profesores muestran sensibilidad ante las diferencias culturales de los alumnos. Sin embargo, se debe crear conciencia sobre la preservación del medio ambiente.
\end{abstract}

Palabras clave: Aprendizaje, Cultura, Organización, Transferencia del Conocimiento

\section{ABSTRACT}

The main objective of this work is to identify, how the organizational and cultural obstacles implied in the transference of knowledge, affect this process in the Faculty of Informatics of the Autonomous University of Queretaro (UAQ). A theoretical analysis on organizational learning, its importance and impact for the transfer of knowledge, was performed, identifying the barriers as a whole. A questionnaire with 126 Likert scale items was applied to 236 undergraduate students. Cronbach's alpha coefficient was .960, indicating a high internal consistency for the items. The confirmatory factor analysis was realized to determine the effect of latent variables on each of the observed variables. Therefore, it was noted that from an organizational view, learning and creativity in educational activities are encouraged by the introduction of new ideas; on the cultural side, teachers are sensitive to cultural differences among students. However, awareness about preserving the natural environment must be raised.

Keywords: Culture, Knowledge Transfer, Learning, Organization

\section{Este artículo se puede referenciar}

Romero, R. (2017). Obstáculos organizacionales y culturales que limitan la transferencia del conocimiento en la Universidad Autónoma de Querétaro. En Desarrollo Gerencial Revista de la Facultad de Ciencias Económicas Administrativas y Contables de la Universidad Simón Bolivar-Colombia, 9(1), 81-96.

\footnotetext{
${ }^{I}$ Este trabajo es producto del proyecto de investigación FOFIUAQ2015 "Factores que limitan la transferencia del conocimiento en el proceso de enseñanza aprendizaje en la Facultad de Informática" con registro FIF201601. Financiado por la Universidad Autónoma de Querétaro. De enero de 2016 a diciembre de 2017.

2 Doctorado en Administración, Profesora-Investigadora, Facultad de Informática, Universidad Autónoma de Querétaro, rossyrg04@yahoo.com.mx.
}

Desarrollo Gerencial, 9 (1) Pp. 81-96 Enero-Junio 2017. ISSN: 2145-5147 (On Line). Universidad Simón Bolívar. Barranquilla-Colombia. Contactos: desarrollogerencial@unisimonbolivar.edu.co 


\section{INTRODUCCIÓN}

De acuerdo con Tinto (1986), en muchas Instituciones de Educación Pública la reprobación y la deserción estudiantil impacta directamente a los indicadores institucionales, menciona que existe un desinterés en los estudiantes durante su paso por una carrera universitaria que puede ser tan elevado que lleva a un abandono de las aulas. Este desinterés puede proceder de diferentes fuentes tales como: problemas de orden afectivo, la falta de integración, necesidades educativas no cubiertas, problemas de auto estima, mala influencia, monotonía en clase por parte de los profesores, pérdida de tiempo en redes sociales, internet, etc. Estos factores impactan directamente en el rendimiento académico, disminución de asistencia y falta de participación en clase, afectando al estudiante a largo plazo en su vida laboral y personal. Por tanto, Sánchez (2014) recomienda que es necesario que el docente realice su trabajo con profesionalismo, que tenga gentilezas que generen la armonía en el aula de clases: que sea inteligente, intuitivo, responsable, optimista, tenaz, benevolente y justo.

Dentro de este contexto, se planteó la pregunta de investigación: ¿Cómo los obstáculos organizacionales y culturales impiden la transferencia del conocimiento en la Facultad de Informática?, en esta perspectiva se plateó el objetivo general para "identificar cómo afectan los obstáculos organizacionales y culturales durante la transferencia del conocimiento". Atendiendo a estas consideraciones, se construyó una encuesta que contiene 126 reactivos, con 5 variables de análisis: tecnología de la información (tecnología); estrategias, métodos y técnicas (procesos); motivación; conocimiento; y, gestión del curso.

\section{ANTECEDENTES TEÓRICOS}

Las universidades representan la sociedad del conocimiento, porque producen y difunden el conocimiento constantemente, son instituciones que se adecúan a entornos competitivos creando vínculos entre la educación, la investigación básica y aplicada. Por tanto, deben ser instituciones que se adapten a un entorno competitivo seleccionando alumnos, profesores, proyectos de investigación de alta calidad. Aunque la enseñanza, la publicación y la divulgación son elementos importantes para la transferencia del conocimiento es necesario identificar los obstáculos que intervienen y que de alguna forma limitan que el conocimiento sea transferido con facilidad no sólo a la comunidad estudiantil, sino también hacia otras instituciones de educación superior.

En una institución educativa, la capacitación y el aprendizaje continuo que requieren los alumnos es de vital importancia, para fomentar el progreso de sus aptitudes y habilidades en busca de un crecimiento personal. Castañeda (2015) asegura que los procesos de formación contribuyen al desarrollo de las competencias, debido a este motivo, Dierkes, Child \& Nonaka (2003) señalan que, a través de la 
transferencia del conocimiento, la institución crece y evoluciona, por lo que, se debe fomentar que el conocimiento de la organización se convierta en experiencias y aprendizaje individual, cuidando que cada alumno tenga acceso y asimile de la mejor forma el conocimiento colectivo necesario.

Para esto, se debe tener en cuenta que el éxito visto desde un enfoque de competitividad en el mercado, debe lograrse por acciones tal como: la comunicación, la capacitación, la constante auditoría; y la evaluación. Todo esto en su conjunto ayuda a optimizar el crecimiento organizacional y el conocimiento individual. En ambos contextos el conocimiento es un factor crítico, por tanto, es importante identificar los obstáculos que limitan el flujo del conocimiento tanto a nivel organizacional como cultural.

Para Planellas \& Muni (2015) el aprendizaje es un círculo continuo y dialéctico, un bucle doble que puede generar conocimiento colectivo, aprendizaje organizacional y estrategias compartidas. Por su parte Villagrasa, Jiménez \& Hernández (2015) lo relacionan con un proceso de adquisición, creación y transformación de conocimiento de forma individual, colectiva e institucional que permite incrementar la capacidad innovadora y competitiva. Para Cyert \& March (1963) es un esquema de ajuste del comportamiento de la organización generado por una respuesta a las variaciones del entorno. Mientras que, Argyris \& Schön (1978) lo definen como un proceso a través del cual "los miembros de una organización detectan errores o anomalías y las corrigen mediante una reestructuración de la teoría de acción sustentada por la organización, integrando los resultados de sus indagaciones en los mapas e imágenes organizacionales" (p. 58). Finalmente, DiBella, Nevis \& Gould (1996) definen el aprendizaje organizacional como "la capacidad dentro de una organización para mantener o mejorar el desempeño basado en la experiencia, a través de la adquisición de conocimiento, el compartir el conocimiento y el uso del conocimiento" (p. 363).

De acuerdo con Senge (1990), el aprendizaje organizacional se debe observar desde un pensamiento sistémico obteniendo una visión integral de la realidad y de la conexión entre los conocimientos, los objetos y situaciones dinámicas. Garzón \& Fisher (2008) subrayan que su filosofía está presente en cada decisión y en cada proceso, e involucra el talento y las competencias de todas las personas. Por todo esto, la transferencia del conocimiento incluye todo tipo de intercambio de conocimiento entre individuos, equipos, grupos y organizaciones. El propósito de la transferencia debe ser claro, muy bien definido y unidireccional; sin embargo, el conocimiento compartido final es multidireccional, informal porque no tiene un objetivo claro y carece de reglas. Argote (1999) señala que la transferencia de conocimiento es la comunicación o el conocimiento de una fuente para que se aprenda y se aplique por parte del receptor. La fuente y el receptor pueden ser individuos, grupos, equipos, unidades organizacionales u organizaciones enteras en cualquier combinación. 
Para Didriksson (2000) la transferencia de conocimiento acerca a la universidad a los sectores productivos y sociales, es por eso que la Organización de las Naciones Unidas para la Educación, la Ciencia y la Cultura (UNESCO, 2009) considera que el proceso de transferencia implica la absorción, adaptación, difusión y reproducción del conocimiento. De acuerdo con González, Clemenza \& Ferrer (2007) en las Instituciones de Educación Superior la transferencia se genera a través del flujo de conocimientos, de publicación de libros, de artículos y otra información publicable; también del intercambio académico de investigadores a nivel internacional; del intercambio de información y de personal dentro de programas de colaboración técnica; a través de contratación de expertos y asesoramiento, por medio de acuerdos; acuerdos de concesión de licencia sobre procedimientos industriales, uso comercial y patentes. Mientras que, para el Ministerio de Economía y Competitividad (2015) se da a través de revistas científicas, libros y presentaciones en congresos, las acciones de transferencia, patentes, internacionalización de las actividades, colaboraciones con grupos nacionales e internacionales y, en su caso, la formación de personal investigador. De esta manera, Crossan, Lane \& White (1999) reconocen que la transferencia del conocimiento se lleva a cabo a través de los procesos institucionalizados en forma de sistemas, estructuras, estrategias y procedimiento.

Todas las formas de transferencia del conocimiento antes señaladas se reflejan de forma importante entre el docente y el alumno en las Instituciones de Educación Superior. De acuerdo con Camelo, García $\&$ Sousa (2010) los docentes realizan acciones que son esenciales en la transferencia del conocimiento para que los alumnos absorban este conocimiento y lo utilicen adecuadamente. Por lo tanto, el aprendizaje individual de los alumnos, de acuerdo con Inkpen \& Crossan (1995) es el resultado de los procesos de aprendizaje, este tipo de aprendizaje lo clasifican en: aprendizaje experimental, aprendizaje forzado, aprendizaje bloqueado, y la ausencia de aprendizaje. Huff (1990) explica que el aprendizaje individual se inicia a partir de la recolección de elementos conscientes; mediante el proceso de interpretación se desarrollan mapas cognoscitivos y el aprendizaje se va integrando mediante metáforas y conversaciones frecuentes. Con este objetivo Ko, Kirsch \& King (2005) consideran que los factores de comunicación son esenciales en estos procesos.

Pawlowski \& Bick (2012) han identificado que existen barreras interpersonales, organizacionales y culturales que inciden en la trasferencia del conocimiento. Paulin \& Suneson (2012) consideran que entre las barreras interpersonales se encuentran la carencia de habilidades, de conocimientos, de seguridad en sí mismo, falta de motivación, falta de confianza, falta de tiempo, intolerancia con los errores.

Por su parte, Borkowski (2009); Peñalva \& Ramírez (2010); March \& Olsen (1976), Argyris (1990), Fahey \& Prusak (1998), McDermott \& O’Dell (2001), CEN (2004), Bick (2004), Riege (2006), y Maier (2007) (mencionados en Pawlowski \& Bick, 2012); y, Baca (2014) identifican un conjuntos de barreras 
organizacionales como son: la falta de liderazgo y dirección empresarial; la escasez de espacios formales e informales para compartir el conocimiento, espacios de reflexión y generación de conocimiento; la falta de recompensas y reconocimiento; cultura corporativa insuficiente; la escasez de infraestructura apropiada; falta de apoyo a las prácticas de intercambio de conocimientos; la deficiencia de recursos de la empresa; restricción de los flujos de comunicación y conocimiento; ambiente de trabajo físico y el diseño de las áreas de trabajo; y, competitividad interna dentro de las unidades de negocio, falta de visión sistémica, ignorar incapacidades y errores, falta de visión de impactos a largo plazo, anclarse en experiencia directa, falta de una actitud proactiva, resistencia al cambio, incapacidad de interpretación y no hacer caso de los procesos ocultos.

En cuanto a las barreras culturales, Pawlowski \& Bick (2012) las identificaron como: la incapacidad de la comunicación y la colaboración, miedo, inseguridad, la falta de conciencia y sensibilidad, la falta de habilidad de integración, falta de voluntad, cuestiones de idioma y el miedo de la imitación. Attewell (1992) agrega la falta de conocimiento tecnológico y su aplicación en el entorno organizacional, así mismo, Filieri (2010) afirma que los factores tecnológicos tienen una mayor repercusión en la correcta transmisión del conocimiento, ya que, mucha información se transmite por estos medios. Por lo que, Hartwick \& Barki (2001) sugieren que las organizaciones deben concentrarse en buscar tecnologías que motiven la relación entre los alumnos y profesores para que compartan sus experiencias y los conocimientos valiosos que poseen.

Además, Carballo (2014) señala que se necesitan espacios para hacer posible la innovación y la creatividad pero se tienen que fomentar los espacios informales, como es el uso de Internet en donde las relaciones tienen un alto contenido enriquecedor; es decir, los ambientes digitales tienen un impacto fundamental en los procesos de transferencia del conocimiento, en este ambiente, se desarrollan espacios de interacción entre las personas (en cualquier parte del mundo), impulsado el intercambio de conocimientos e información. Aunado a esto, Bueno (1995) menciona que cuando se reconoce el esfuerzo, la mejora y los logros individualmente de los alumnos a través de recompensas, premios y reconocimientos se promueven los progresos individuales.

\section{MÉTODO}

\section{$\checkmark$ Diseño}

Este estudio se realizó bajo el paradigma cuantitativo, es un estudio transversal porque se realizó en un punto específico del tiempo, durante el mes de mayo del 2016 en los programas de licenciatura de la Facultad de Informática de la Universidad Autónoma de Querétaro. Es un estudio descriptivo porque representa la frecuencia de una población definida. El criterio de inclusión se fundamentó tomando en cuenta las características de los programas académicos del objeto de estudio vinculados con el objetivo de 
la investigación, por tanto, se incluyeron a todos los alumnos de nivel licenciatura, excluyendo a los alumnos de maestría y doctorado, debido a lo anterior el tamaño de la población es de 594 alumnos.

\section{$\checkmark$ Participantes}

Se aplicaron 236 encuestas a una población de 594 alumnos, al calcular el tamaño de la muestra con un nivel del 95\% de confianza se indicó que se debían aplicar 234. Durante el muestreo se seleccionaron muestras aleatorias simples de alumnos de cada licenciatura de los cuales el $7.6 \%$ fueron estudiantes de la Licenciatura en Administración en Tecnologías de Información, el 15.3\% de la Licenciatura en Informática, el 14.4\% de Ingeniería en Computación, el 46.6\% de Ingeniería en Software y finalmente el 16.1\% de Ingeniería en Telecomunicaciones y Redes. En la Tabla 1 se observa la participación de los alumnos por semestre, los alumnos de cuarto tuvieron una participación del 30\%, los alumnos de primer semestre del $16.9 \%$ y los alumnos del sexto semestre del $16.9 \%$.

Tabla 1. Escolaridad y edad

\begin{tabular}{lcclcc}
\hline \multicolumn{1}{c}{ Semestre } & $\boldsymbol{\%}$ & $\boldsymbol{F}$ & \multicolumn{1}{c}{ Edad } & $\boldsymbol{\%}$ & $\boldsymbol{f}$ \\
\hline Cuarto Semestre & 30.9 & 73 & de 20 a 21 años & 37.3 & 88 \\
Primer Semestre & 16.9 & 40 & de 18 a 19 años & 30.5 & 72 \\
Sexto Semestre & 16.9 & 40 & de 22 a 23años & 22.0 & 52 \\
Segundo Semestre & 15.7 & 37 & de 28 a 30 años & 3.8 & 9 \\
Octavo Semestre & 5.5 & 13 & de 26 a 28 años & 3.4 & 8 \\
Quinto Semestre & 4.2 & 10 & de 24 a 25 años & 2.1 & 5 \\
Séptimo Semestre & 3.8 & 9 & de 15 a 17 años & .4 & 1 \\
Tercer Semestre & 3.4 & 8 & mayor de 35 años & .4 & 1 \\
Noveno Semestre & 2.5 & 6 & & &
\end{tabular}

Fuente: Elaboración propia (2016).

De los 236 participantes el $82.6 \%$ fueron hombres mientras que el $17.4 \%$ fueron mujeres, se observó también que el $93.2 \%$ son solteros (as), el $4.2 \%$ vive en unión libre, el $1.3 \%$ está divorciado(a) y finalmente el $.8 \%$ es casado (a).

\section{$\checkmark$ Instrumentos}

La encuesta utilizada contiene 126 reactivos, se construyó con 5 variables de análisis: tecnología de la información (tecnología); estrategias, métodos y técnicas (procesos); motivación; conocimiento; y gestión del curso. Se calculó el alfa de cronbach para determinar el grado de consistencia interna de las escalas que permite medir la confiabilidad del instrumento realizado bajo la escala Likert con escalas de 1 a 5, este coeficiente fue de .960 de acuerdo con George \& Mallery (1995) y Schmitt (1996) señalan que si el alpha 
de cronbach es mayor que 0.9 el instrumento de medición es excelente. Al aplicar la prueba de Kolmogorov - Smirnov se observó que los datos tenían una distribución normal ( $p \geq .05)$.

\section{$\checkmark$ Procedimiento}

En un inicio se observó el problema existente entre la forma en como los estudiantes de la Facultad han tenido problemas al recibir el conocimiento transferido por sus profesores, por lo que se decidió analizar esas barreras y así fortalecer el aprendizaje de los alumnos. Por tanto, después de analizar una serie de posibles factores se diseñó una encuesta que contiene 126 reactivos y se construyó con 5 variables de análisis: tecnología de la información (tecnología); estrategias, métodos y técnicas (procesos); motivación; conocimiento; y gestión del curso. Se procedió a la validación de los datos para identificar si existían anomalías procedentes de la captura. A continuación, se calculó el alfa de cronbach para determinar el grado de consistencia interna de las escalas que permite medir la confiabilidad del instrumento realizado bajo la escala Likert con escalas de 1 a 5.

\section{RESULTADOS}

Para identificar las barreras organizacionales se definieron las variables de: estrategias, métodos y técnicas (procesos); motivación; y, conocimiento. Para identificación de las barreras culturales se analizó la influencia de la tecnología de la información (tecnología); la gestión del curso explorando el ambiente para compartir el conocimiento y valores que se transmiten en el aula.

\subsection{Estrategias, métodos y técnicas (espacios de reflexión y generación de conocimiento)}

El aula es un espacio lugar de aprendizaje compartido, un espacio de reflexión y generación de conocimiento es un punto de intercambio de visiones, experiencias y prácticas que mejoran el acercamiento entre el docente y el alumno al realizar reflexiones compartidas y experiencias personales, es un espacio de interacción continua que genera constantemente conocimiento e impulsa que el estudiante haga su propia autorreflexión.

En esta variable se alcanzó un Alpha de Cronbach de .822, en el análisis de factorial confirmatorio se obtuvo un índice $K M O$ con un valor de .859 y la prueba de esfericidad de Bartlett $\left(\mathrm{Ji}_{234}^{2}=487.87 ; \mathrm{p}^{<}\right.$ .0001) indica que la muestra es muy adecuada para efectuar la factorización de los ítems. Por tanto, se efectuó el análisis factorial y durante la extracción a través del análisis de componentes principales, con el método de rotación varimax con Kaiser (Tabla 2), se generó solamente un componente que obtuvo un autovalor mayor de uno. 
Tabla 2. Matriz de componentes rotados para estrategias, métodos y técnicas (espacios de generación y reflexión del conocimiento)

\begin{tabular}{lc} 
& Componente \\
\cline { 2 - 2 } & 1 \\
\hline 42. Durante el semestre se establecen estrategias de aprendizaje & .762 \\
30. ¿Se fomenta un debate continuo sobre los temas explicados con información interna y & .759 \\
$\quad$ externa? & .725 \\
35. En clase, tus profesores propician que reflexiones sobre tu aprendizaje & .721 \\
23. ¿Proponen actividades formativas en clase? & .707 \\
45. Consideran los resultados de las evaluaciones para proponer mejoras en el aprendizaje & .602 \\
48. Tus profesores son abierto para corregir errores de apreciación y evaluación & .602 \\
24. ¿Escuchan todas las opiniones sugeridas? &
\end{tabular}

Fuente: Elaboración propia (2016).

En los espacios de reflexión y generación de conocimiento se observó que el $73.3 \%$ de los alumnos indican que sus profesores escuchan todas las opiniones sugeridas en clase, el $72.5 \%$ indican que son abierto para corregir errores de apreciación y evaluación, el $66.5 \%$ señala que durante el semestre se establecen estrategias de aprendizaje. El $65.7 \%$ indican que sus profesores consideran los resultados de las evaluaciones para proponer mejoras en el aprendizaje. El $63.3 \%$ opinan que en clase se proponen actividades formativas, el $60.2 \%$ opinan que se propicia que el alumno reflexiones sobre su aprendizaje. Finalmente, el $59.3 \%$ establece que se fomenta un debate continuo sobre los temas explicados con información interna y externa.

\subsection{Motivación a través de recompensas y reconocimiento}

La dimensión de motivación alude al uso formal e informal de recompensas, incentivos y alabanzas. El tipo de recompensas, las razones para darlas y la distribución de las mismas, tiene importantes consecuencias para el desarrollo del interés para aprender en los alumnos y provee sentimientos de autoeficacia y satisfacción por su aprendizaje. En esta variable se obtuvo un Alpha de Cronbach de .836, al efectuar el análisis de factorial confirmatorio se obtuvo un índice $K M O$ con un valor de .852 y la prueba de esfericidad de Bartlett $\left(\mathrm{Ji}_{234}{ }_{234}=471.73 ; \mathrm{p}<\right.$.0001) indica que la muestra es muy adecuada para la factorización de los ítems. Por tanto, se llevó a cabo el análisis factorial y la extracción a través del análisis de componentes principales, con el método de rotación varimax con Kaiser (Tabla 3).

En este análisis se seleccionaron dos componentes porque de acuerdo a la gráfica de segmentación representan el $67 \%$ de la varianza total acumulada, debido a que el Criterio de Kaiser indica que se deben conservar los criterios que tengan un autovalor mayor que uno, aunque el criterio más utilizado es observar el criterio de varianza total explicada por cada componente o factor y cuando normalmente se acerca al $80 \%$ significa que ese número de factores es suficiente. El componente 1 (recompensas) está 
integrado los ítems 49, 51 y 52, mientras que en el componente 2 (reconocimiento) se agruparon los ítems 17,54 y 56.

Tabla 3. Matriz de componentes rotados motivación (recompensas y reconocimientos)

\begin{tabular}{lcc}
\hline & \multicolumn{2}{c}{ Componente } \\
\cline { 2 - 3 } & 1 & 2 \\
\hline 49. ¿Toman en cuenta tu esfuerzo realizado durante el curso? & $\mathbf{. 8 3 5}$ & .184 \\
51. Promueven la inquietud por aprender & .753 & .323 \\
52. Reconocen tus logros cuando propones una idea nueva & .744 & .300 \\
17. ¿Existen incentivos para el aprendizaje en clase? & .131 & $\mathbf{. 8 5 8}$ \\
54. Hacen que te sientas interesado en la materia & .455 & $\mathbf{. 6 3 6}$ \\
56. ¿Consideras que tu participación en clase fue valorada? & .397 & $\mathbf{. 6 8 5}$ \\
\hline
\end{tabular}

Fuente: Elaboración propia (2016)

En el componente de recompensas se observó que el $66.1 \%$ de los alumnos que participaron en la encuesta indica que se toman en cuenta los esfuerzos realizados durante el curso, el $62.3 \%$ están de acuerdo o totalmente de acuerdo en que se promueve la inquietud por aprender. El $63.1 \%$ señalan que sus profesores reconocen los logros cuando proponen alguna nueva idea.

Para el componente de reconocimientos, el $52.1 \%$ indican que existen incentivos para el aprendizaje en clase, mientras que el $47.5 \%$ no está de acuerdo ni en desacuerdo, o en desacuerdo o totalmente en desacuerdo sobre la existencia de incentivos para el aprendizaje. El $61 \%$ señalan que sus profesores despiertan el interés por su materia. Finalmente, el $70.3 \%$ indican que fue valorada su participación en clase.

\subsection{Conocimiento (prácticas de intercambio de conocimientos)}

En el intercambio de conocimientos, ideas, pensamientos, emociones y sentimiento es lo que realmente genera un nuevo conocimiento, implica habilidades y destrezas para establecer una comunicación pedagógica efectiva y eficiente con los alumnos. Se origina a través de la socialización del conocimiento tácito en sesiones de creatividad, grupos de trabajo, enseñanza de maestros a aprendices; generalmente los grupos y equipos son los protagonistas de estos intercambios. En este intercambio, el alumno aprende a través de la interrelación con sus compañeros, maestros y demás personas de su entorno.

En esta variable se obtuvo un Alpha de Cronbach de .905, en el análisis de factorial confirmatorio se obtuvo un índice $K M O$ con un valor de .931 y la prueba de esfericidad de Bartlett $\left(\mathrm{Ji}_{234}^{2}=1222.46\right.$; $p<.0001)$ indica una que es una excelente muestra para efectuar la factorización de los ítems. Por tanto, al realizar la extracción a través del análisis de componentes principales, con el método de rotación varimax con Kaiser (Tabla 4) se obtuvieron dos componentes de acuerdo al Criterio de Kaiser. El componente 1 (intercambio de conocimientos) está integrado por los ítems 39, 7, 11, 18, 16, 22, 9 y 6; mientras que el componente 2 (dominio de la materia) contiene a los ítems 10, 14, 12, 8 y 15. 
Tabla 4. Matriz de componentes rotados de conocimiento (apoyo a la práctica de intercambio de conocimientos)

\begin{tabular}{lcc}
\hline & \multicolumn{2}{c}{ Componente } \\
\cline { 2 - 3 } & 1 & 2 \\
\hline 39. Se producen discusiones en donde participe todo el grupo & .693 & .063 \\
7. ¿Buscan un punto de vista común propiciando la participación de los alumnos & $\mathbf{. 6 7 4}$ & .288 \\
en clase? & $\mathbf{. 6 7 2}$ & .317 \\
11. ¿Se fomenta la creatividad siendo receptivo a nuevas ideas? & $\mathbf{. 6 4 4}$ & .311 \\
18. ¿Se comunica de una forma clara y fácil de entender? & $\mathbf{. 6 0 8}$ & .315 \\
16. ¿Se generan soluciones a través de la resolución de problemas reales? & $\mathbf{. 5 9 9}$ & .400 \\
22. ¿Te brindan ayuda académica cuando requieres intercambio de & $\mathbf{. 5 7 8}$ & .304 \\
conocimiento? & $\mathbf{. 5 4 8}$ & .471 \\
9. ¿Existe comprensión en la forma de entender y pensar del grupo? & & \\
6. ¿Tus profesores, comparten las mejores prácticas utilizando ejemplos útiles & .220 & $\mathbf{. 8 3 6}$ \\
para explicar la asignatura? & .229 & $\mathbf{. 7 8 2}$ \\
10. ¿Consideras que tus profesores poseen un conocimiento avanzado de su & .247 & $\mathbf{. 6 4 4}$ \\
asignatura? & .375 & $\mathbf{. 6 4 2}$ \\
14. ¿Dominan los contenidos de la materia? & .391 & $\mathbf{. 6 2 5}$ \\
12. ¿Existe comprensión y respeto para los alumnos de parte de los profesores? & &
\end{tabular}

Fuente: Elaboración propia (2016).

En el componente de intercambio de conocimientos entre los alumnos y docentes se observó que: el $59.3 \%$ está de acuerdo y totalmente de acuerdo en que en clase se generan discusiones en donde participa todo el grupo. El $71.6 \%$ dicen que sus profesores propician la participación de los alumnos buscando un punto de vista común. El $61.4 \%$ exterioriza que sus profesores fomentan la creatividad siendo receptivos a nuevas ideas. El $64 \%$ expresan que se comunican de forma clara y fácil de entender. El $69.1 \%$ especifican que se generan soluciones a través de problemas reales. El $80 \%$ de los alumnos encuestados señalan que sus profesores les ayudan académicamente cuando requieren intercambio de conocimientos. Para el $58 \%$ existe comprensión en la forma de pensar del grupo. Finalmente, para este componente, el $66.1 \%$ indica que, para explicar la materia, los profesores utilizan problemas reales.

En el componente de dominio del docente en la materia se observó que: $80.9 \%$ consideran que sus profesores poseen un conocimiento avanzado en la materia y el $81.7 \%$ exterioriza que dominan su contenido. El $86 \%$ subraya que existe comprensión y respeto para los alumnos. Por su parte el $71.6 \%$ señalan que se comparten los conocimientos en clase integrando la teoría con la práctica. Finalmente, el 74.6\% especifica que sus profesores están actualizados sobre los avances de su asignatura.

\subsection{Tecnología de la información (espacios formales e informales para compartir el conocimiento)}

En esta variable se obtuvo un Alpha de Cronbach de .821, en el análisis factorial confirmatorio se realizó la prueba de esfericidad de Bartlett $\left.\left(\mathrm{Ji}^{2}{ }_{234}=623.302 ; p<.0001\right)\right)$ y el valor índice $K M O$ obtuvo un valor de .791, de acuerdo con Ximénez \& San Martín (2013) este indicador se encuentra dentro de los 
límites establecidos, por lo que es adecuado el uso del análisis factorial. Durante la extracción a través del análisis de componentes principales, con el método de rotación varimax con Kaiser (Tabla 5), se indicó que las preguntas 66, 67, 68 y 72 se agruparan el componente 1 (Tecnologías), mientras que los ítems 70, 71 y 69 se agruparan en el componente 2 (Comunicación). Al analizar el Alpha de Cronbach el proceso sugirió no tomar en cuenta el ítem 27 que se refiere al espacio físico ya que este ítem formaba un clúster muy distante de los otros componentes.

Tabla 5. Matriz de componentes rotados para tecnologías de la información como espacios formales e informales para compartir el conocimiento

Componente

1 2

67. Promueven el uso de diversas herramientas, particularmente las digitales, para gestionar (recabar, procesar, evaluar y usar) información.

.881 .162

66. Tus profesores utilizan las tecnologías de la información como un medio que facilite el aprendizaje de los estudiantes

68. Promueven el uso seguro, legal y ético de la información digital

72. Utilizan plataformas especiales para promover el aprendizaje

70. Utilizan correos electrónicos para impulsar el aprendizaje

71. Utilizan redes sociales para fomentar el aprendizaje

69. Explican un concepto o tema con dispositivos audiovisuales $\mathbf{8 5 4} \quad .191$

$.743 \quad .158$

$.602 \quad .511$

$.063 \quad .860$

$.198 \quad .811$

$.351 \quad .512$

Fuente: Elaboración propia (2016).

En el componente de tecnologías se observó que el $85.2 \%$ están de acuerdo o totalmente de acuerdo que los profesores promueven el uso de diversas herramientas tecnológicas, particularmente digitales, para gestionar (recabar, procesar, evaluar y usar) información. El $86.4 \%$ están de acuerdo o totalmente de acuerdo que sus profesores utilizan las tecnologías de la información como un medio para facilitar el aprendizaje. El $82.2 \%$ aseguran que se promueve el uso seguro, legal y ético de la información digital. Mientras que el $66.1 \%$ de los participantes indican que sus profesores utilizan plataformas especiales para promover el aprendizaje.

En cuanto a la comunicación como espacio informal para transferir el conocimiento se observó que: El $80 \%$ indica que sus profesores utilizan correos electrónicos como medio de comunicación para impulsar el aprendizaje. Para el uso de redes sociales se observó que solamente el $44.9 \%$ las utiliza para fomentar el aprendizaje, mientras que el $36 \%$ no está de acuerdo ni en desacuerdo, y solamente el 18.6\% está en desacuerdo o totalmente en desacuerdo que se utilizan las redes sociales como estrategia de comunicación. Finalmente, para el uso de dispositivos audiovisuales se observó que el $86.4 \%$ señala que sus profesores explican un concepto o tema con el uso de estos dispositivos. 


\subsection{Gestión del curso}

En esta variable se obtuvo un Alpha de Cronbach de .805, al efectuar el análisis de factorial confirmatorio obtuvo un índice $K M O$ con un valor de .805 y la prueba de esfericidad de Bartlett $\left(\mathrm{Ji}_{234}^{2}=541.12 ; p<.0001\right)$, todo esto indica que la muestra es muy adecuada para realizar la factorización de los ítems. Por tanto, al efectuar la extracción a través del análisis de componentes principales, con el método de rotación varimax con Kaiser (Tabla 6) se generaron dos componentes. En el componente 1 (Ambiente para compartir el conocimiento) están integrado los ítems 29, 26, 25, 28, 13, y 34, mientras que en el componente 2 (Valores) se agruparon los ítems 20, 19 y 21.

Tabla 6. Matriz de componentes rotados para cultura

\begin{tabular}{lcc}
\hline & \multicolumn{2}{c}{ Componente } \\
\cline { 2 - 3 } & \multicolumn{1}{c}{1} & \multicolumn{1}{c}{2} \\
\hline 29. ¿Consideras que las relaciones interpersonales en tu clase son de armonía? &. $\mathbf{7 5 5}$ & .116 \\
26. ¿Fomentan la creatividad y la introducción de nuevas ideas? & $\mathbf{. 7 3 1}$ & .243 \\
25. ¿Tienen tolerancia con los errores cometidos en las actividades de clase, &. $\mathbf{6 9 3}$ & .072 \\
aprovechándolos para aprender? & $\mathbf{. 6 1 7}$ & .297 \\
28. ¿Explican los temas de clase con situaciones de la vida real? & $\mathbf{. 6 0 6}$ & .337 \\
13. ¿Tus profesores demuestran sensibilidad ante las diferencias culturales? & $\mathbf{. 5 1 9}$ & .065 \\
34. ¿Se propicia que seas tú el responsable de tu propio aprendizaje? & .155 & $\mathbf{. 7 8 5}$ \\
20. ¿Fomentan la importancia de contribuir a la conservación del medio ambiente? & .069 & $\mathbf{. 7 7 2}$ \\
19. ¿Tus profesores generalmente asisten a clases de forma regular y puntual? & .415 & $\mathbf{. 5 9 3}$ \\
21. ¿Se promueve mantener limpias y ordenadas las instalaciones? & &
\end{tabular}

Fuente: Elaboración propia (2016).

En el componente que conforma el ambiente para compartir el conocimiento se observó que: el 80 $\%$ de los alumnos que participaron opinan que en el aula de clase las relaciones interpersonales son de armonía, el $67 \%$ considera que se fomenta la creatividad y se introducen ideas nuevas, de esta forma, el $75 \%$ reconoce que los errores que existen en clase durante las actividades educativas se aprovechan para aprender. En este sentido, $65.7 \%$ valora que se expliquen los temas con situaciones de la vida real. Así mismo, se observó que los profesores muestran sensibilidad ante las diferencias culturales, ya que el $81 \%$ está de acuerdo y totalmente de acuerdo con este concepto. Por su parte, el $72.9 \%$ aprecia que se propicie que el alumno sea responsable de su propio aprendizaje

Para el componente de valores, el $39.4 \%$ no está de acuerdo ni en desacuerdo que se fomente la importancia de contribuir a la conservación del medio ambiente, aunque el $34.7 \%$ está de acuerdo y totalmente de acuerdo se hace énfasis en esta importancia. Mientras que el $73.3 \%$ consideran que se promueve mantener limpias y ordenadas las instalaciones, finalmente en cuanto a la puntualidad de los profesores el $60.6 \%$ de los alumnos encuestados consideran que asisten a clase en forma regular y puntual. 


\section{DISCUSIÓN}

El objetivo principal de este trabajo fue identificar cómo afectan las barreras organizacionales y culturales en la transferencia del conocimiento en la Facultad de Informática. En las barreras organizaciones mencionadas por Borkowski (2009); Peñalva \& Ramírez (2010); March \& Olsen (1976), Argyris (1990), Fahey \& Prusak (1998), McDermott \& O’Dell (2001), CEN (2004), Bick (2004), Riege (2006), y Maier (2007) (mencionados en Pawlowski \& Bick, 2012); y, Baca (2014), se tomaron en cuenta los siguientes aspectos: los espacios formales e informales para compartir el conocimiento, que mejoren la reflexión y generación de conocimiento; la falta de recompensas y reconocimiento; la escasez de infraestructura apropiada; falta de apoyo a las prácticas de intercambio de conocimientos; y, restricción de los flujos de comunicación y conocimiento. Se observó que:

Es importante que en la Facultad de Informática se fomenten los espacios de reflexión y generación de conocimiento a través de un debate continuo sobre los temas explicados con información interna y externa, que se impulse al alumno sobre la reflexión de su aprendizaje, que se promuevan actividades formativas, también es recomendable que se consideren los resultados de las evaluaciones para establecer estrategias y, proponer mejoras en el aprendizaje.

En cuanto a recompensas y reconocimiento, se sugiere que: los profesores den incentivos en clase para que el alumno se sienta motivado en la materia, se promueva la inquietud por aprender, se reconozcan los logros del estudiante cuando proponga ideas innovadoras, y sobre todo, se resalte el esfuerzo del estudiante durante el curso.

En la variable de apoyo a las prácticas de intercambio de conocimientos, aunque resultó muy bien evaluada se debe reforzar que los profesores promuevan las discusiones grupales, impulsen la creatividad siendo receptivos a nuevas ideas, se comuniquen de una forma clara y fácil de entender, generen soluciones a través de problemas reales compartiendo las mejores prácticas para explicar la materia. Finalmente, comprendan la forma de entender y pensar del grupo.

Las barreras culturales identificadas por Attewell (1992) y Pawlowski \& Bick (2012) están integradas por: falta de conocimiento tecnológico y su aplicación en el entorno organizacional, la gestión del curso explorando el ambiente para compartir el conocimiento y los valores que se transmiten. Dentro de este contexto, se observó que en la Facultad de Informática de la UAQ el uso de la tecnología ha tenido un factor relevante; sin embargo, se recomienda promover el uso de las redes sociales como estrategia de transferencia del conocimiento e impulsar el uso de las plataformas especiales

En el ambiente para compartir el conocimiento y los valores que se trasmiten, se detectó que en el aula de clase las relaciones interpersonales son de armonía, que se fomenta la creatividad y se introducen ideas nuevas, se reconocen que los errores que existen en clase durante las actividades educativas 
aprovechándolos para aprender, los profesores muestran sensibilidad ante las diferencias culturales, propician que el alumno sea responsable de su propio aprendizaje, promueven mantener limpias y ordenadas las instalaciones; y, que los profesores asisten a clase en forma regular y puntual

No obstante, se propone que los profesores complementen su práctica docente con la conservación del medio ambiente a través de actividades que involucren a los alumnos, que se promuevan valores, actitudes, habilidades y una participación activa totalmente organizada.

\section{6. - REFERENCIAS}

Argote, L. (1999). Organizational learning: Creating, retaining and transferring knowledge. Boston: Kluwer Academic Publishers.

Argyris, C. \& Schön, D. A. (1978). Organizational learning: a theory of action perspective. USA: Addison-Wesley.

Attewell, P. (1992). Technology diffusion and organizational learning - the case of business computing. Organization Science. 3(1). pp 1-19. Recuperado de: http://pubsonline.informs.org/doi/abs/10.1287/orsc.3.1.1

Baca, U. G. (2014). Administración integral hacia un enfoque de procesos. México: Grupo Editorial Patria.

Borkowski, N. (2009). Organizational Behavior in Health Care. Estados Unidos: Jones \& Bartlett Publishers.

Bueno, A. J. A: (1995). Motivación. Programas de intervención. Beltrán, Ll. J. \& Bueno, A. J. A. (Eds.). Psicología de la educación. España: Editorial Boizareu Universitaria Marcombo.

Camelo, C., García, J. \& Sousa, E. (2010). Facilitadores de los procesos de compartir conocimiento y su influencia sobre la innovación. Cuadernos de Economía y Dirección de la Empresa. (42). 113-150.

Carballo, R. (2014). Innovación y gestión del conocimiento. Madrid: Ediciones Díaz de Santos.

Castañeda, D. I. (2015). Condiciones para el aprendizaje organizacional. Estudios Gerenciales. 31(134). 62-67. Recuperado de: http://www.redalyc.org/articulo.oa?id=21233043007

Crossan, M. M., Lane, W. H. \& White, E. R. (1999). An Organizational Learning Framework: from Intuition to Institution. Academy of Management Review. 24(3). 522-537.

Cyert, R. M. \& March, J. (1963). A Behavioral Theory of the Firm. Englewood Cliffs. NJ: Prentice-Hall.

DiBella, A., Nevis, E. C. \& Gould, J. M. (1996). Understanding organizational learning capability. Journal of Management Studies. 33. 361-379. 
Didriksson, A. (2000). La universidad del futuro: relaciones entre educación superior, la ciencia y la tecnología. México: Plaza y Valdés.

Dierkes, M., Child, J. \& Nonaka, I. (2003). Handbook of Organizational Learning and Knowledge. Reino Unido: Oxford University Press.

Filieri, R. (2010). Overcoming Knowledge Sharing Barriers through Communities of Practice: Empirical Evidence from a Big Automotive Supplier. Reino Unido: Cambridge Scholars Publishing.

Garzón, C. M. A. \& Fisher, A. L. (2008). Modelo teórico de aprendizaje organizacional. Pensamiento y Gestión (24). 195-224. Recuperado de: http://www.redalyc.org/articulo.oa?id=64602408

George, D. \& Mallery, P. (1995). SPSS/PC+ Step-by-step: a simple guide and introduction. California: Wadsworth Publishing Company.

González, V., Clemenza, C. \& Ferrer, J. (2007). Vinculación universidad-sector productivo a través del proceso de transferencia de tecnología. Revista de Estudios interdisciplinarios en Ciencias Sociales. 9 (2). Recuperado de: http://www.redalyc.org/pdf/993/99318750006.pdf

Hartwick, J., \& Barki, H. (2001). Interpersonal conflict and its management in information system development. MIS Quarterly. 25(2). 195-228.

Huff, A. S. (1990). Mapping Strategic Thought. UK: Wiley

Inkpen, A. \& Crossan, M. (1995). Believing is seeing: Organizational leaming in joint ventures. Journal of Management Studies. 32(5). 595-618.

Ko, D. G., Kirsch, L. J., \& King, W. R. (2005). Antecedents of knowledge transfer from consultants to clients in enterprise system implementations. Management Information System Quarterly, 29(1), $59-85$.

Ministerio de Economía y Competitividad (2015). Boletín Oficial del Estado, 3 (149). Recuperado de: https://www.boe.es/boe/dias/2015/06/23/pdfs/BOE-A-2015-6983.pdf

Paulin, D., \& Suneson, K. (2012). Knowledge transfer, knowledge sharing and knowledge barriers-three blurry terms in KM. The Electronic Journal of Knowledge Management, 10(1), 81-91.

Pawlowski, J. M., \& Bick, M. (2015). The global knowledge management framework: Towards a theory for knowledge management in globally distributed settings. Lead. Issues Knowl. Manag. Vol. Two, 2, 134. 


\begin{tabular}{|c|c|}
\hline 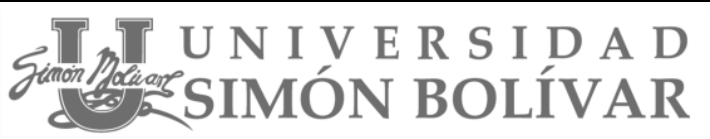 & $\begin{array}{l}\text { Desarrollo Gerencial } \\
\text { Revista de la facultad de ciencias } \\
\text { economicas adminintstativas y contables. }\end{array}$ \\
\hline
\end{tabular}

Peñalva, R. L. P. \& Ramírez, A. H. T. (2010). Barreras para el aprendizaje organizacional Estudio de caso método. Producción $2007 . \quad$ Reconómica de: http://148.206.107.15/biblioteca_digital/capitulos/377-5224ltb.pdf

Planellas, M. \& Muni, A. (2015). Las decisiones estratégicas: los 30 modelos más útiles. Barcelona: CONECTA

Sánchez, C. F. A. (2014). El docente frente al reto de motivar al alumno. Revista Iberoamericana Producción Académica y Gestión Educativa. Recuperado de: https://www.pag.org.mx/index.php/PAG/article/view/134/182

Schmitt, N. (1996). Uses \& abuses of coefficient Alpha. Psychological Assessment, 8 (4), 350 - 353.

Senge, P. (1990). The Fifth Discipline: The Art and Practice of The Learning Organization. Estados Unidos: Crown Publishing Group.

Tinto, V. (1986). Una reconsideración de las teorías de la deserción estudiantil. En trayectoria escolar en la educación superior. México: ANUIES-SEP

UNESCO (2009). World Conference on Higher Education: The New Dynamics on Higher Education and Research For Societal Change and Develoment. Paris: UNESCO

Villagrasa, A., Jiménez, M. \& Hernández, J. (2015). Implicaciones del aprendizaje organizacional en la pequeña y mediana empresa de Cacao-Sucre. Negotium, 11(31), 24-47. Recuperado de: http://www.redalyc.org/pdf/782/78241171003.pdf

Ximénez, G. M. C. San Martín, C. R. (2013). Fundamentos de las técnicas multivariantes. España: UNED. Universidad Nacional de Educación a Distancia. 